\title{
Enormous Ovarian Borderline Serous Cystic Fibroma in Postmenopausal Patient. A Case
}

\author{
Sofoudis Chrisostomos ${ }^{1 *}$, Moschopoulou Sevasti ${ }^{1}$, Apessou Dimitra ${ }^{2}$, Manes Konstantinos ${ }^{3}$, Fagrezos \\ Dimitrios $^{4}$, Karouta Afroditi ${ }^{5}$ and Gerolymatos Andreas ${ }^{1}$ \\ ${ }^{1}$ Department of Obstetrics and Gynecology, Konstandopoulio General Hospital Athens, Greece \\ ${ }^{2}$ Department of Pathology, Konstandopoulio General Hospital Athens, Greece \\ ${ }^{3}$ Department of Surgery, Konstandopoulio General Hospital Athens, Greece \\ ${ }^{4}$ Department of Computed Tomography, Konstandopoulio General Hospital Athens, Greece \\ ${ }^{5}$ Department of Ultrasound, Konstandopoulio General Hospital Athens, Greece
}

*Corresponding author: Chrisostomos Sofoudis, Department of Obstetrics and Gynecology, Konstandopoulio General Hospital Athens, Ippokratous 209, 11472, Athens, Greece.

Received Date: August 05, 2019

Published Date: August 12, 2019

\section{Abstract}

Ovarian serous cystadenomas are benign tumors that arise from ovarian epithelium. They represent the commonest type accounting for approximately $60 \%$.

They affect all ages with a peak incidence between the 4th and 5th decade of life. Their definite differentiation from other ovarian masses is based on histological findings. Even though serous cystadenomas are quite commonly diagnosed ovarian masses, the diagnosis of unusually enormous masses is still quite rare.

This case report refers to the case of a post-menopausal 52-year-old woman, admitted at our Department, complaining of abdominal swelling over the past 3 years. An abdominal CT scan performed, revealing an enormous mass, originating from the right ovary, extending throughout the whole abdominal cavity towards the diaphragm up to the xiphoid process. Patient underwent exploratory laparotomy revealing an ovarian mass with median diameter $30 \times 25 \times 15 \mathrm{~cm}$.

She underwent a total abdominal hysterectomy with bilateral salpigoopherectomy and dissection of the momentum, as part of surgical staging of the lesion. Final histopathological report confirmed the details of the frozen biopsy, announcing the presence of serous cystic fibroma of borderline malignancy.

Multidisciplinary approach proposed follow up of the patient with assiduous imaging findings.

Keywords: Ovarian mass; Borderline malignancy; Cystic fibroma

\section{Introduction}

Diagnosis of ovarian masses consists a very common finding in Gynecology and in some cases may even be spontaneous in completely asymptomatic patients. Due to final histopathologic evaluation, they can be benign, malignant or borderline [1].

Depending on tumors origin are divided into germ cell, stromal and epithelial tumors. Epithelial tumors represent the majority of all types [2]. Among epithelial tumors serous cystadenomas consist the commonest type [3].

Clinical presentation of patients suffering from an ovarian serous cystadenoma can be non-specific and varying. While the commonest symptom is pelvic pain or discomfort, patients who have larger masses may present with a painless distended abdominal cavity.

In some cases, the lesion be completely asymptomatic and may represent an incidental finding on pelvic ultrasound or CT scan of the pelvis.

Performing pelvic ultrasound serous cystadenomas are seen as unilocular anechoic cystic adnexal lesions, without papillary projections or blood flow on Doppler scanning. Some may be multilocular. On abdominal CT scan these masses are seen as cystic 
with homogeneous CT attenuation, with a thin regular wall or septum, and usually no endocystic or exocystic vegetation. Tumor markers including Ca125 are not specific concerning the diagnosis of ovarian serous cystadenomas.

Surgical mapping of ovarian cysts depends on tumors size, imaging findings and tumors markers range.

Final diagnosis is based on histological findings which typically consist of solitary layers of benign epithelial cells.

We hereby present the case of a 52-year-old post-menopausal female nurse, diagnosed with a rarely enormous ovarian mass, histopathologically identified as an ovarian serous cystic-fibroma borderline malignancy.

Our case report aims at studying and emphasizing the importance of early and proper diagnosis of such ovarian masses [4] as well as the post-operative management of these patients.

\section{Case}

We present a case of a 52-year-old female nurse (para 0, gravida 0) admitted at our Department complaining of episodes of abdominal distention.

In terms of symptoms apart from the obvious abdominal distention and feeling of heaviness, she reported no other clinical manifestation.

There were any episodes of gastrointestinal disturbance or abdominal pain. Her abdominal cavity had been distending over the past 3 years. The patient herself had initially attributed the abdominal distension to weight gain and consulted several dieticians.

Her medical history was unremarkable, with no serious illnesses in the past. In terms of surgical history, she had undergone tonsillectomy as a child and thyroidectomy at the age of 30 due to a benign thyroid mass followed by appropriate pharmaceutical agents.

Free obstetrical history reaching menopause at the age of 50 . She reported no allergies and had no family history of malignant disease whatsoever.

Clinical examination revealed presence of palpable mass extending the whole peritoneal cavity up to the xiphoid process. The mass was painless on palpation. There were no palpable lymph nodes. Palpation of the liver or the spleen was impossible. Bowel sounds were present. There was no other clinical evidence.

Her pre-operative blood tests including renal and liver function tests were normal with the exception of a slightly elevated Ca-125 at 58.54 with normal range of 35.

She was initially referred for an abdominal and pelvicultrasound scan, which revealed a huge cystic mass originating from the right ovary and extending up to the diaphragm (Figure 1).

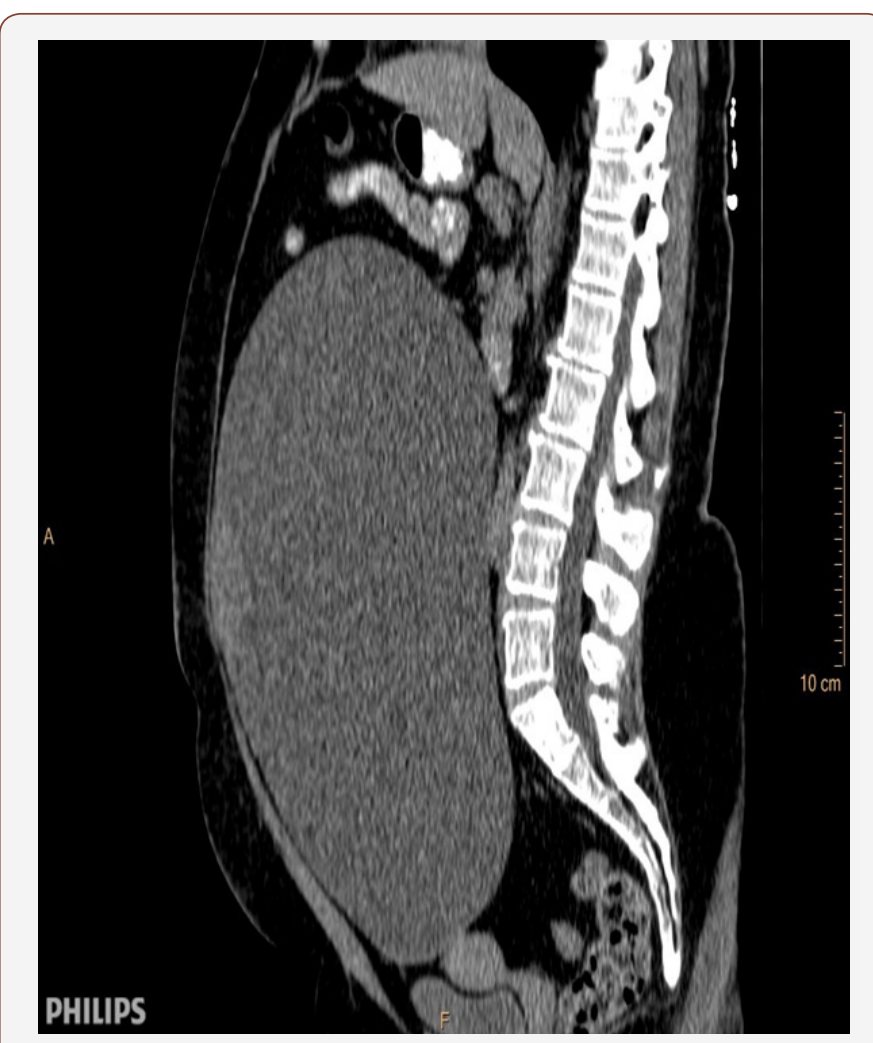

Figure 1: Enormous ovarian borderline serous cystic fibroma. Abdominal CT scan.

She performed an abdominal and pelvic CT, revealing presence of a massive, mainly cystic mass measuring $23 \times 26 \times 16 \mathrm{~cm}$ with two areas of high density. The mass was seen to be in close proximity to the uterus and the possible origination from the right ovary was confirmed on the CT scan.

As result of all preoperative mapping, patient underwent total abdominal hysterectomy and bilateral salpingooopherectomy with surgical dissection of the momentum.

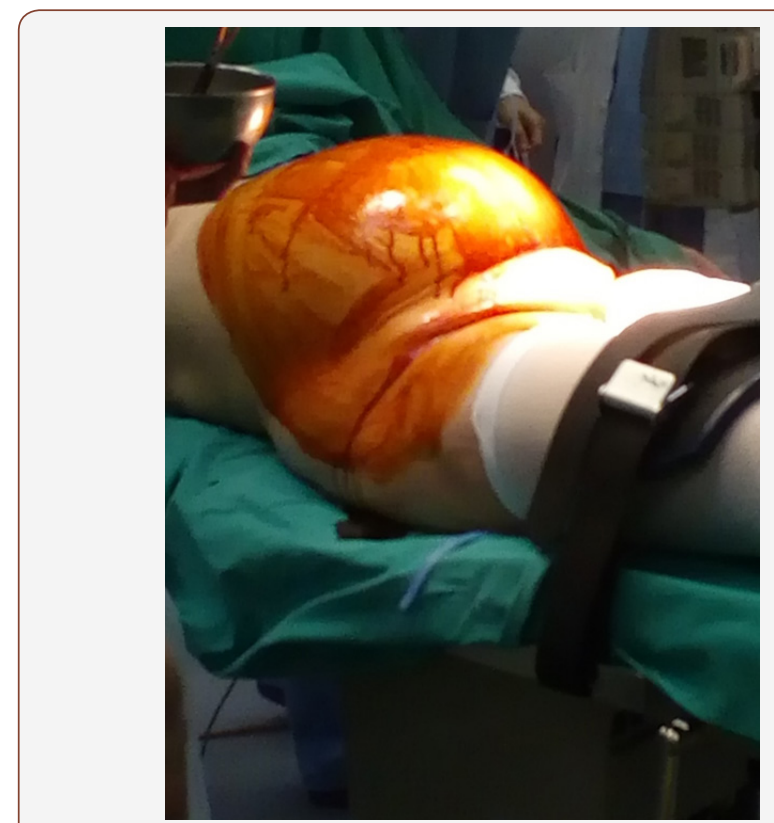

Figure 2: Operative surgical preparation. 
Intra-operatively following a vertical abdominal midline incision extending above and below the umbilicus, an enormous cyst was removed intact, originating from the right ovary. The cyst was sent for frozen biopsy, revealing a borderline malignancy mass most probably representing a serous cystadenoma. Surgical result, dissection of the momentum as well (Figures $2 \& 3$ ).

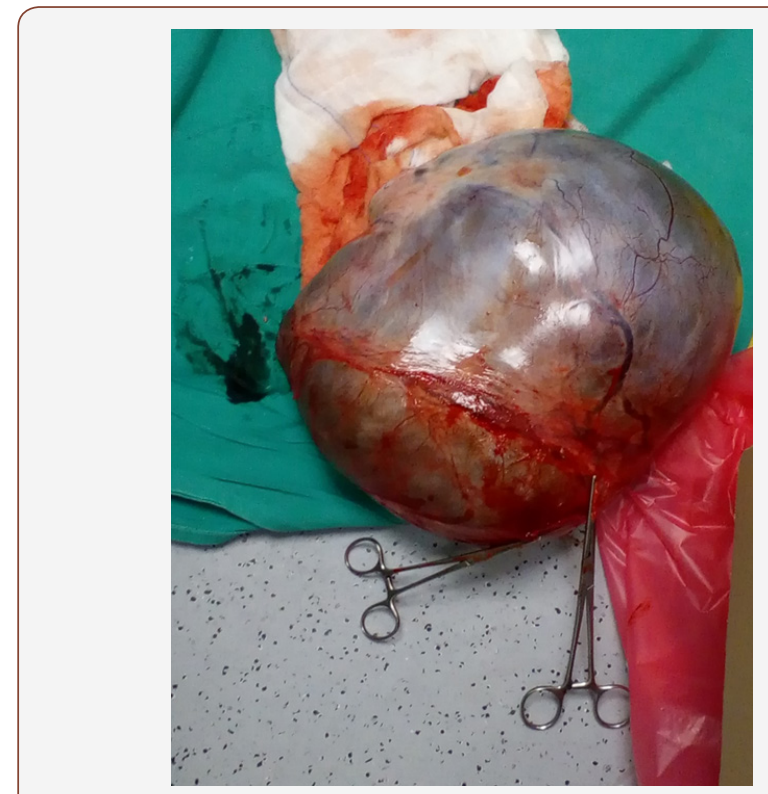

Figure 3: Enormous borderline ovarian cystic fibroma.

A drainage tube was inserted in the pouch of Douglas. There was free fluid in the abdomen which was aspirated and sent for cytology. There was any sigh of peritoneal malignancy.

Macroscopically the resected mass measured $30 \times 25 \times 15 \mathrm{~cm}$, had a smooth surface with two areas of smooth extending nodules measuring around $5 \mathrm{~cm}$ each. The mass was macroscopically identified as a unilocular cystic fibroma filled with cystic fluid. However, there were identified areas with histological features of borderline malignancy.

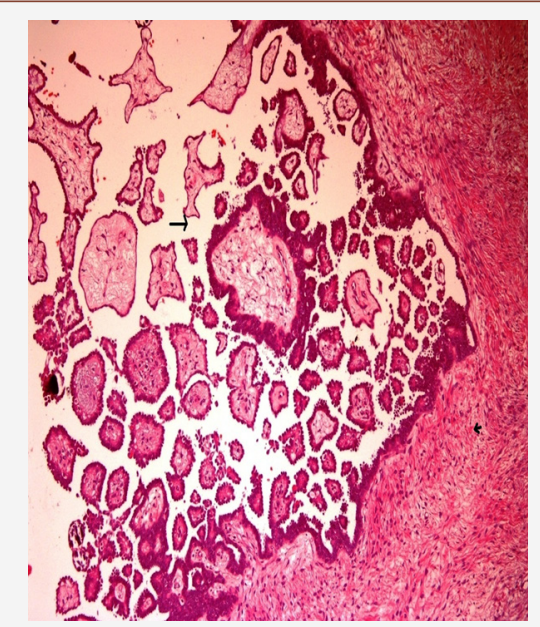

Figure 4: Epithelial hyperplasia site: Small arrow: fibrous layer, Large arrow: Epithelial hyperplasia, microtubule formation and stagnation. Because in many tested histological sections this morphology was observed in a single focal area less than $10 \%$ of the lesion, the neoplasm is classified as a serous cystadenoma with focal epithelial hyperplasia. H\&E X 200.
Therefore, ovarian serous cystadenomas expressed with less than $10 \%$ morphology were identified as serous cystadenomas with local epithelial hyperplasia (Figures 4-6).

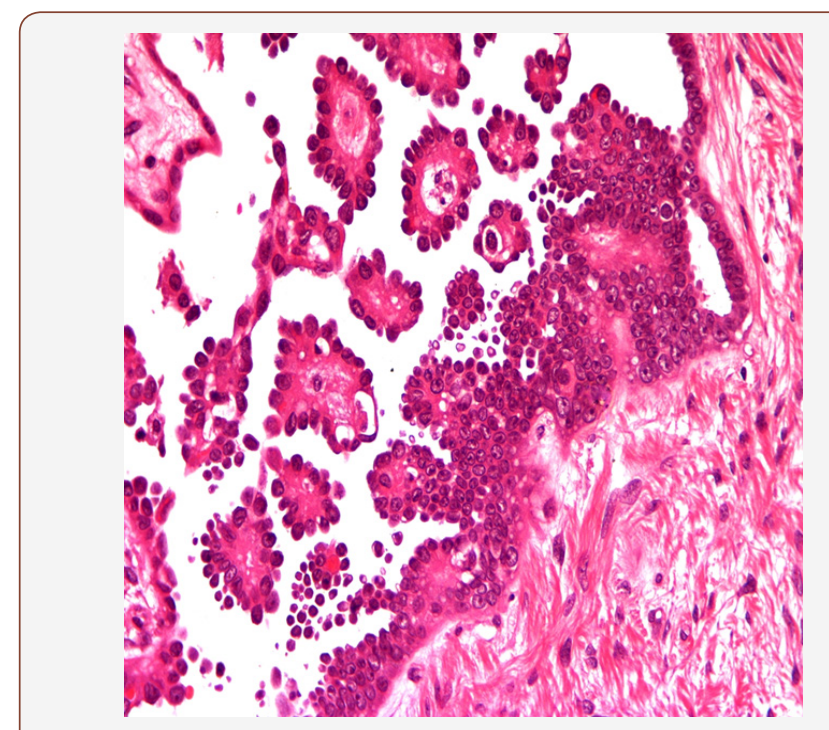

Figure 5: Epithelial hyperplasia area in higher magnification. H\&E X400.
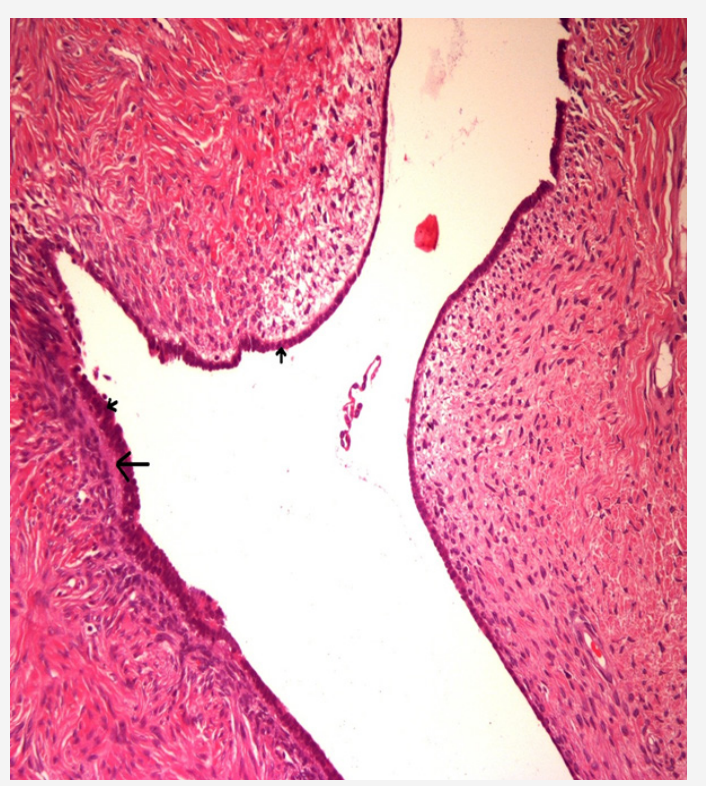

Figure 6: Throughout the rest, the cystic neoplasm had typical histological characteristics of a benign serous cystic fibroma. Small arrows: Epithelium consists of one to a few lines of cylindrical cells without atypia. Large arrow: typical subcellular zone subepithelial. H\&E X 200.

During 1st pod clinical status of paralytic ileus was established, diagnosed through clinical examination and abdominal CT. Management mapping of paralytic ileus was conservative.

Huge amounts of ascitic fluid were drained from the drainage tube, which was removed on the 4 th pod. Patient was discharged from the hospital in good clinical condition in the 6th pod.

Multidisciplinary approach decided to follow up of the patient with assiduous imaging findings, with no need for episodes of chemotherapy or radiotherapy at present. 


\section{Discussion}

As mentioned above the diagnosis of ovarian cystic masses is extremely common with epithelial tumors comprising about half of all ovarian tumors [2].

Benign serous tumors of all subtypes account for about 25\% of all benign ovarian neoplasms. About $10 \%$ of them are bilateral, while $70 \%$ of them are benign and only $5-10 \%$ of them have borderline malignant potential [2].

Back in 1922 Spohn reported a serous cyst adenoma that weighed 148.6kg, while later on in 1963 Symmonds reported a serous cystadenoma weighing $79.4 \mathrm{~kg}$.

In 2009 Vellanki, et al [2] published the case of a woman from whom a serous cystadenoma measuring $60 \times 47 \times 30 \mathrm{~cm}$ was dissected.

Therefore, the size of the ovarian mass, the histologic mixed type along with its borderline malignant potential consist our case rare and worth reporting.

Even though the patient was a health professional, the delay in diagnosing such case depended on the fact, that she underestimated and misinterpreted the symptom of severe and ongoing abdominal distention she experienced over the past 3 years. The absence of other symptoms also contributed to the delay of diagnosis.

Serous cystadenomas carry a very good prognosis with very low potential of recurrence. According to recent bibliography, ultimate goal remains, follow up of the patient with assiduous imaging findings.

\section{Conclusion}

Even though ovarian cystic masses are very common they are usually diagnosed at an early stage.

Occasionally the absence of specific clinical manifestations as well as the personal insight of the patient towards any non-specific symptoms may result in a significant delay in the diagnosis.

Our goal remains proper diagnosis and treatment, with ultimate scope the increase of the quality of life of the patient.

\section{Acknowledgement}

None.

\section{Conflict of Interest}

Authors declare no conflict of interest.

\section{References}

1. Ross EK, Kebria M (2013) Incidental ovarian cysts: When to reassure, when to reassess, when to refer. Cleve Clin J Med 80(8): 503-514.

2. Sujatha VV, Babu SC (2009) Giant ovarian serous cystadenoma in a postmenopausal woman: a case report. Cases J 2: 7875.

3. Mülayim B, Gürakan H, Dagli V, Mülayim S, Aydin O, Akkaya H (2006) Unaware of a giant serous cyst adenoma: a case report. Arch Gynaecol Obstet 273(6): 381-383.

4. Guideline on the Management of Ovarian Masses. Norfolk and Norwich University Hospitals NHS Trust, UK. 